\title{
Gerbard Lozek
}

\section{Die deutsche Geschichte 1917/18 bis 1945 in der For- schung der DDR (1945 bis Ende der sechziger Jahre)}

Der Geschichtsabschnitt von $1917 / 18$ bis 1945 fand in der Geschichtswissenschaft der DDR von Anfang an besondere Aufmerksamkeit aufgrund seiner höchst aktuellen, unmittelbar in das Gegenwartsgeschehen hineinwirkenden historischen Erfahrungen vor allem zweier von Deutschland ausgegangener Weltkriege. Die spezifischen Probleme der Forschung, Lehre und Publikation zu dieser historischen Periode sind jedoch - wie zu anderen partiellen Entwicklungen auch - nur im Zusammenhang mit der Gesamtentwicklung der DDR-Geschichtswissenschaft und ihrer unmittelbaren Vorgeschichte zu verstehen. Das war bekanntlich ein Aufbruch zu neuen Ufern, ein Aufbruch, dessen geistige Voraussetzungen allerdings schon vor 1945 vor allem im antifaschistischen Wirken der KPD und anderer demokratischer Kräfte geschaffen worden waren ${ }^{1}$.

Die Geschichtswissenschaft der DDR durchlief bis Ende der 60er Jahre drei Entwicklungsphasen: von 1945 bis zu Beginn der 50er Jahre die Phase ihrer Grundlegung; in den 50er Jahren die Phase ihrer eigentlichen Konstituierung; in den 60er Jahren die Phase ihrer Konsolidierung, in der sich ihre normalen „Reproduktionsmechanismen" entfalten und schließlich dauerhaft wirksam werden konnten ${ }^{2}$. Dies gilt im wesentlichen auch für den hier interessierenden geschichtswissenschaftlichen Teilbereich. Daraus resultiert aber auch, daß das im Generalthema des Kolloquiums als Zāsur gesetzte Jahr 1965 für die Forschungen zur deutschen Geschichte von 1917/18 bis 1945 in der Historiographiegeschichte der DDR keinen Entwicklungseinschnitt darstellt; dieser Einschnitt liegt Ende der 60er Jahre.

Die historiographiegeschichtliche Forschung der DDR hat die genannten Etappen bislang noch unterschiedlich aufgearbeitet. Für die erste Etappe beschränken sich die bisherigen Untersuchungen auf den Neubeginn 1945/46 sowie auf einige Studien zur

\footnotetext{
${ }^{1}$ Vgl. Werner Bertbold, Marxistisches Geschichtsbild - Volksfront und antifaschistisch-demokratische Revolution (Berlin 1970) 27-155; im folgenden zitiert: Bertbold, Marxistisches Geschichtsbild.

${ }^{2}$ Vgl. Werner Berthold, Zur Entwicklung der marxistisch-leninistischen Geschichtswissenchaft zu einer voll entfalteten wissenschaftlichen Spezialdisziplin, in: Beiträge zur Geschichte der Arbeiterbewegung (im folgenden: BzG) 26 (1984) 13-24; Walter Schmidt, Zur Geschichte der DDRGeschichtswissenschaft vom Ende des zweiten Weltkrieges bis zur Gegenwart, in: BzG 27 (1985) 614-633.
} 
Universitätsgeschichte ${ }^{3}$. Am gründlichsten erforscht sind die 50er Jahre ${ }^{4}$. Noch im Anfangsstadium stehen die Studien $\mathrm{zu}$ den $60 \mathrm{er}$ Jahren. Eine allgemeine Orientierungsgrundlage bieten die anläßlich der Welthistorikerkongresse in Stockholm (1960), Moskau (1970) und Bukarest (1980) veröffentlichten Sonderbānde der „Zeitschrift für Geschichtswissenschaft ${ }^{*}$, die thematisch gegliederte Ubersichten zu den historischen Forschungen in der DDR im jeweils vorangegangenen Jahrzehnt enthalten.

Im Zeitraum von 1945 bis Anfang der 50er Jabre, der Grundlegungsphase der marxistischen Geschichtswissenschaft, gab es noch keine systematischen Forschungen zur deutschen Geschichte nach 1918. Nach der Entfernung aller aktiven Träger der faschistischen Ideologie aus dem Schul- und Hochschulwesen sowie anderen wissenschaftlichen Einrichtungen lag das Schwergewicht auf der Erarbeitung und Einführung neuer Lehrprogramme ${ }^{5}$, die sich konsequent mit den Wurzeln und dem Wesen des deutschen Faschismus auseinandersetzten, die Schwächen der Weimarer Republik verdeutlichten und sich auf das Vermächtnis des antifaschistischen Widerstandes hin orientierten.

Dies geschah im engen Zusammenwirken von Marxisten und Nichtmarxisten. Im akademischen Wissenschaftsbetrieb dominierten in jenen Jahren die nichtmarxistischen Historiker. Die meisten von ihnen vertraten einen von bürgerlich-humanistischen Úberzeugungen geprāgten antifaschistischen Standpunkt. Einige waren in der Folgezeit nicht bereit, den neuen gesellschaftlichen Weg mitzugehen, sie verließen zumeist die sowjetische Besatzungszone. Viele aber blieben und nahmen tatkräftig an der Neugestaltung des Bildungswesens und des Wissenschaftsbetriebes teil; sie leisteten Bedeutendes beim Aufbau geschichtswissenschaftlicher Institute, Archive und Bi-

${ }^{3}$ Vgl. Berthold, Marxistisches Geschichtsbild, 156-205; Werner Mägdefrau, Zum Kampf um eine neue Geschichtswissenschaft an der Friedrich-Schiller-Universitāt Jena, in: WZ der FSU Jena 15 (1966) 63-77; Günter Katsch, Zur Entwicklung der Geschichtswissenschaft an der Karl-MarxUniversitāt Leipzig, in: WZ der KMU Leipzig 31 (1982) 544-558; Christian Christ-Thilo, Die Etablierung der Wirtschaftsgeschichte als eine maxistisch-leninistische Gesellschaftswissenschaft an der Wirtschaftswissenschaftlichen Fakultāt der Berliner Universitāt durch Jürgen Kuczynski (1946-1956), in: Beiträge zur Geschichte der Humboldt-Universitāt zu Berlin 10 (1984) 9-56.

4 Die Entwicklung der DDR-Geschichtswissenschaft im angegebenen Zeitraum ist Gegenstand von fünf Dissertations- und zwei Habilitationsschriften: Helmut Heinz über die Phase von Mitte 1950 bis Mitte 1952 (Berlin 1977), im folgenden zitiert: Heinz, DDR-Geschichtswissenschaft 1950/52; Horst Haun über die Phase von Mitte 1952 bis Anfang 1954 (Berlin 1978); Uwe Fischer zur Rolle des Autorenkollektivs für das Lehrbuch der Geschichte des deutschen Volkes. 1952 bis Mitte 1955 (Berlin 1981), im folgenden zitiert: Fischer, Autorenkollektiv 1952/55; Horst Helas zur Rolle dieses Autorenkollektivs von Mitte 1955 bis Mitte 1958 (Berlin 1985), im folgenden zitiert: Helas, Autorenkollektiv 1955/58; Carola Seiler zur Herausbildung der Geschichte der Arbeiterbewegung als eigenständige Teildisziplin in der zweiten Hälfte der 50er Jahre (Berlin 1986), im folgenden zitiert: Seiler, Geschichte der Arbeiterbewegung; Horst Haun zur Entstehung und Gründung der Deutschen Historiker-Gesellschaft (Berlin 1984); Helmut Heinz zu den Lehrbriefen „Geschichte“ des ersten Fernstudiums für Geschichtslehrer von 1952 bis 1954 (Berlin 1985). Eine Studie zur Genesis der zeitgeschichtlichen Forschung vermittelt Heinz Heitzer, „Zeitgeschichte" 1945 bis 1958, in: ZfG 35 (1987) 99-115.

${ }^{5} \mathrm{Vgl}$. Berthold, Marxistisches Geschichtsbild, 206-258. 
bliotheken, wirkten bei der Heranbildung des wissenschaftlichen Nachwuchses mit und waren auch zur wissenschaftlichen Kooperation mit den anfangs wenigen marxistischen Fachkollegen bereit. Stellvertretend seien hier genannt: Otto Hoetzsch (1876-1946) und Fritz Rörig (1882-1953) in Berlin, Heinrich Sproemberg (1889-1966) zuerst in Rostock und später in Leipzig, Eduard Winter (1896-1982) in Halle, Karl Griewank (1900-1953) zunächst in Berlin, dann wie Friedrich Schneider (1887-1962) und Hugo Preller (1887-1968) in Jena.

Für die spāteren Forschungen zur deutschen Geschichte zwischen 1917/18 und 1945 hatten die zum Teil schon in der Emigration entstandenen Publikationen von namhaften Zeitzeugen hohen Wert, so von Albert Norden ${ }^{6}$, Wilhelm Pieck ${ }^{7}$ und Otto Buchwitz $^{8}$. Eine breite Wirkung erzielte die unmittelbar nach dem Kriegsende veröffentlichte Schrift Walter Ulbrichts „Die Legende vom ,deutschen Sozialismus" “9, die das Wesen des Faschismus enthüllte.

Das herausragende Werk zur deutschen Geschichte seit dem 16.Jahrhundert, das in zwei Kapiteln auf die Zeit nach 1918 einging, war in jenen Jahren zweifellos Alexander Abuschs „Der Irrweg einer Nation. Ein Beitrag zum Verstāndnis deutscher Geschichte ${ }^{\text {“10 }}$. Dieses Buch übte eine außerordentlich positive Rolle bei der Herausbildung eines antifaschistisch-demokratischen Bewußtseins aus. Seine Wirkung erklārte sich nicht schlechthin aus der besonders akzentuierten Kennzeichnung der reaktionāren Kräfte in der deutschen Geschichte, sondern zugleich aus der engagierten Darstellung ihrer Gegenkräfte, auch des antifaschistischen Widerstandes, den Abusch in seiner ganzen Breite faßte als „eine moralische Kampfgemeinschaft von den Kommunisten und Sozialdemokraten bis zu katholischen Pfarrern und konservativen Demokra$\operatorname{ten}^{\text {“11. }}$.

In dem Bestreben, die Ursachen für negative Seiten der deutschen Geschichte bewußt zu machen, was in der gegebenen Situation besonders notwendig war, kam es mitunter zu einer Überhöhung dieser Entwicklungen. Zugleich wandte sich Abusch aber unmißverstāndlich gegen eine fatalistische Sicht der Vergangenheit mit der programmatischen Feststellung: „Mögen einst alle Wege nach Rom geführt haben, nicht alle deutschen Wege mußten unvermeidlich zu Hitler führen." ${ }^{12}$

Langfristig wirkende Forschungsimpulse für die Geschichte der Weimarer Republik gingen von dem geschichtsträchtigen Jahr 1948 aus, dem 100.Jahrestag der Revolution von 1848/49 sowie dem 30.Jahrestag der deutschen Novemberrevolution von 1918. Stimulierenden Einfluß auf die konzeptionellen Debatten zu beiden Ereignissen hatten thematische Stellungnahmen des Parteivorstandes der Sozialistischen Einheits-

\footnotetext{
${ }^{6}$ Albert Norden, Lehren deutscher Geschichte. Zur politischen Rolle des Finanzkapitals und der Junker (Berlin 1947).

7 Wilbelm Pieck, Zur Geschichte der Kommunistischen Partei Deutschlands (Berlin 1949).

${ }^{8}$ Otto Bucbwitz, 50 Jahre Funktionär der deutschen Arbeiterbewegung (Berlin 1949).

${ }^{9}$ (Berlin 1945).

${ }^{10}$ (Mexiko 1945, Berlin 1946).

${ }^{11}$ Alexander Abusch, Der Irrweg einer Nation (Berlin $\left.{ }^{2} 1947\right) 263$.

12 Ebenda, 204.
} 
partei Deutschlands ${ }^{13}$. Daraus entstand die erste nach 1945 veröffentlichte marxistische Darstellung über die Novemberrevolution ${ }^{14}$.

Einen weiteren Schwerpunkt der hier interessierenden Periode bildete die Entstehungs- und Wirkungsgeschichte der Weimarer Verfassung. Ungeachtet ihrer Mängel, vor allem des verhängnisvollen Artikels 48, wurde betont, daß diese Verfassung wesentliche Ansātze für die Schaffung eines demokratischen Staatswesens enthalten habe $^{15}$. Eine herausragende Forschungsleistung in der unmittelbaren Nachkriegsphase waren die sozial- und wirtschaftsgeschichtlichen Arbeiten Jürgen Kuczynskis ${ }^{16}$.

Der Durchbruch zur Neugestaltung der Geschichtswissenschaft erfolgte in den 50er Jabren, der eigentlichen Konstituierungsphase als marxistische Wissenschaftsdisziplin. Diese Entwicklung verlief widerspruchsvoll und konfliktreich, dominierend war jedoch stets ein Geist des schöpferischen Suchens und Vorwärtsdrängens. Eine Reihe von Grundproblemen der deutschen Geschichte, die neue Antworten erheischten, wurden im produktiven Meinungsstreit und oft auch in echter Gemeinschaftsarbeit geklārt. Ein hervorstechendes Merkmal des Jahrzehnts waren theoretisch-methodologische und konzeptionelle Debatten, die letztlich von der allgemeinen Zielsetzung geprägt waren, in absehbarer Zeit marxistische Gesamtdarstellungen zur deutschen Geschichte sowie zur Geschichte der deutschen Arbeiterbewegung auszuarbeiten.

Diese Zielvorgabe war nach eingehenden Beratungen mit Historikern vom Zentralkomitee der SED im Oktober 1951 beschlossen worden ${ }^{17}$. Mit Festlegungen zum beschleunigten Aufbau des Museums für Deutsche Geschichte und der Einrichtung von Instituten zur Geschichte des deutschen Volkes an den Universitäten Berlin, Leipzig und Halle sowie eines Instituts für deutsche Geschichte an der Deutschen Akademie der Wissenschaften in Berlin wurden zugleich die institutionellen, materiellen und personellen Voraussetzungen für die Lösung der gestellten Aufgaben geschaffen.

Der erwāhnte Beschluß regte zugleich an, ein mehrbändiges „Lehrbuch der Geschichte des deutschen Volkes“ auszuarbeiten ${ }^{18}$. Im Juni 1952 fand eine erste zentrale Historikerkonferenz der DDR mit über 200 Teilnehmern statt, auf der seine Gestaltung beraten wurde ${ }^{19}$. Bald darauf konstituierte sich ein Autorenkollektiv von zehn namhaften Historikern, das sich auf die anlaufenden Forschungen und Zuarbeiten vieler geschichtswissenschaftlicher Institutionen stützen konnte. Die Periode von 1918

${ }^{13}$ Dokumente der Sozialistischen Einheitspartei Deutschlands, Bd. II (Berlin 1952) 100-116; im folgenden zitiert: Dokumente.

${ }^{14}$ Otto Grotewobl, Dreißig Jahre später. Die Novemberrevolution und die Lehren der Geschichte der deutschen Arbeiterbewegung (Berlin 1948).

${ }^{15} \mathrm{Vgl}$. Karl Polak, Die Weimarer Verfassung, ihre Errungenschaften und Māngel (Berlin 1948).

${ }^{16}$ Jüngen Kuczynski, Die Bewegung der deutschen Wirtschaft von 1800-1946 (Berlin 1946); ders., Die Geschichte der Lage der Arbeiter in Deutschland, Bd. 1: 1800-1932 (Berlin 1947); ders., Studien zur Geschichte des deutschen Imperialismus, Bd. I und II (Berlin 1948, 1950).

${ }^{17}$ Dokumente, Bd. III (Berlin 1952) 581-583.

${ }^{18}$ Die publizierte Fassung lautete: Lehrbuch der deutschen Geschichte (Beiträge) 1-12 (Berlin 1959-1969).

${ }_{19}$ Vgl. Helmut Heinz, Die erste zentrale Tagung der Historiker der DDR 1952, in: ZfG 26 (1978) 387-399. 
bis $1945^{20}$ hatten zunāchst Albert Schreiner (1892-1979) und Erich Paterna (1897-1982) übernommen.

Es ging um eine neue Art, Geschichte zu schreiben, nämlich auf der Grundlage des historischen Materialismus. Von den vielfältigen inhaltlichen Problemen, die zu klären waren, soll hier nur auf die zu Beginn der 50er Jahre geführte Auseinandersetzung um die sogenannten Misere-Auffassungen zur deutschen Geschichte verwiesen werden ${ }^{21}$. Im Grunde genommen handelte es sich bei diesen Auffassungen um eine Variante des "deutschen Sonderweges“. Die Führung der KPD hatte bereits in der zweiten Hälfte der 30er Jahre dieses Problem gründlich behandelt und so beantwortet, daß die deutsche Geschichte trotz ihrer negativen Seiten, die - wie zuletzt 1933 - durch die Handlungen reaktionärer Kräfte geprägt waren, keinesfalls eine Misere-Entwicklung darstelle, sondern - wie die Geschichte anderer Völker auch - ihre positiven Seiten und progressiven Traditionen enthalte ${ }^{22}$. Anton Ackermann, führender Funktionär der KPD, erklārte in diesem Zusammenhang 1937 zur Geschichte der Weimarer Republik: „Die Ursache der Mängel und Fehler der Weimarer Republik ist nicht in ... einer speziellen ,deutschen Eigenart' zu suchen.“23

Eine wichtige Ausgangsposition für eine marxistische Gesamtsicht der deutschen Geschichte wurde mit der Eröffnung des Museums für deutsche Geschichte im Sommer 1952 und seiner ersten Ausstellung erreicht, die auch eine Abteilung 1918-1945 enthielt. Dem Museum war ein Wissenschaftlicher Rat beigeordnet, in den zahlreiche nichtmarxistische Historiker berufen worden waren. Gerhard Ritter nahm das zum Anlaß, um sich anmaßend einzumischen und brieflich die DDR-Mitglieder des Verbandes der Historiker Deutschlands aufzufordern, die Zusammenarbeit mit Marxisten aufzukündigen $^{24}$. Leider befolgte dies ein Teil jener Historiker.

Mitte der 50er Jahre wurde in der seit 1953 erscheinenden „Zeitschrift für Geschichtswissenschaft" eine öffentliche Diskussion der Lehrbuchdisposition zur Periode von $1917 / 18$ bis 1945 begonnen, die die ganze zweite Hälfte der 50er Jahre anhielt. Dazu wurden Gutachten mit kontroversen Meinungen veröffentlicht ${ }^{25}$. Inhaltliche Schwerpunkte der Diskussion, die in der Folgezeit maßgeblich die geschichtswissenschaftlichen Forschungen initiierten und beeinflußten, waren:

${ }^{20}$ Albert Schreiner, Disposition des Hochschullehrbuches der Geschichte des deutschen Volkes (1918-1945), in: ZfG 2 (1954) 701-758.

21 Vgl. Heinz Kamnitzer, Keine "Geschichte der deutschen Misere“, in: Forum 1 (1952); Dokumente, Bd. III, 570-588; Lothar Bertbold, Zur Geschichte der nationalen Konzeption der deutschen Arbeiterklasse, in: ZfG 11 (1963) 5-28.

22 Vgl. Berthold, Marxistisches Geschichtsbild, 32-64.

${ }^{23}$ Zitiert nach Berthold, Marxistisches Geschichtsbild, 59.

${ }^{24}$ Rundschreiben von Gerbard Ritter an die Ausschußmitglieder des VDH vom 1.4.1952 und Protokoll der Ausschußsitzung des VDH vom 24.9.1952, in: Nachlaß Karl Griewank (Universitātsbibliothek der Friedrich-Schiller-Universitāt Jena) Karton 4.

${ }^{25}$ Vgl. Gutachten von W. J. Brjunin, Walter Schmidt und anderen in: ZfG 3 (1955) 243-275; die Stellungnahme dazu von Albert Scbreiner, Zu einigen Fragen der neuesten deutschen Geschichte, in: Ebenda, 374-430. 
Erstens - Periodisierungsfragen. Ein Hauptproblem betraf die Epochenzāsur. Zunehmend wurde nach dem Zusammenhang zwischen der Oktoberrevolution 1917 in Rußland und der Novemberrevolution 1918 in Deutschland gefragt. Obgleich der epochale Einschnitt 1917 für die allgemeine Geschichte unumstritten war, galt für die deutsche Geschichte noch geraume Zeit der Einschnitt November $1918^{26}$. Erst 1958 einigten sich die Autoren des Lehrbuches, die Zäsur 1917 als den Beginn einer neuen Epoche in der deutschen Geschichte anzusehen ${ }^{27}$.

Klärende Debatten gab es weiterhin vornehmlich über den Beginn und das Ende der Novemberrevolution, über den Beginn und die Etappen des Faschisierungsprozesses am Ende der Weimarer Republik sowie über die Tiefe der Einschnitte 1933, 1939 und 1945. Die Errichtung der faschistischen Diktatur 1933 wurde als eine tiefgreifende qualitative Verānderung des Geschehens gewertet, ohne dabei deren Epochenzusammenhang zu übersehen. Ab 1958 setzte sich schließlich in der Geschichtswissenschaft der DDR die folgende Periodisierung des Zeitraumes von 1917 bis 1945 in sieben Teilabschnitten durch: Oktober 1917 bis Oktober 1918; Oktober 1918 bis Januar 1919 (dieser Einschnitt verschob sich später auf Mai 1919); Januar 1919 bis Ende 1923; Anfang 1924 bis Mitte 1929; Mitte 1929 bis Januar 1933; Januar 1933 bis August 1939; September 1939 bis Mai $1945^{28}$.

Zweitens - Probleme der Novemberrevolution sowie die Rolle der Răte in der Revolution. Das Kernproblem betraf die historische Einordnung der Novemberrevolution, was die Frage nach dem Charakter der Revolution aufwarf. Es galt zu klāren, ob die Novemberrevolution als eine bürgerliche, bürgerlich-demokratische oder als eine niedergeschlagene proletarische Revolution zu charakterisieren war. Diese Fragestellung war nicht neu, sie wurde bereits in den 20er und 30er Jahren in der KPD und auch in der Kommunistischen Internationale diskutiert und 1938/39 in dem Sinne beantwortet, die Novemberrevolution als eine bürgerlich-demokratische Revolution zu bewerten $^{29}$. Diese Einschätzung korrespondierte mit der von der Berner Konferenz der KPD nach dem Sturz Hitlers angestrebten "neuen demokratischen Republik“, die den negativen Erfahrungen der Weimarer Republik entgegengesetzt sein und die bürgerlich-demokratische Revolution unter antifaschistischen Vorzeichen zu Ende führen sollte $^{30}$. Mit dem Versuch, die Novemberrevolution als eine gescheiterte proletarische Revolution zu charakterisieren, gingen einige DDR-Historiker hinter den Erkenntnisstand von 1939 zurück ${ }^{31}$.

${ }^{26} \mathrm{Vgl}$. Ebenda; Walter Schmidt, Wilbelm Webling, Bemerkungen zur Disposition für das Hochschullehrbuch der Geschichte des deutschen Volkes (1918-1945), in: ZfG 3 (1955) 256-265.

${ }^{27} \mathrm{Vgl}$. Helas, Autorenkollektiv 1955/58, 121-141. Zunächst vehement abgelehnt, fand diese Periodisierung ein Vierteljahrhundert spāter auch in der Bundesrepublik Verbreitung; zum Beispiel Hagen Schulze, Weimar. Deutschland 1917-1933 (Die Deutschen und ihre Nation 4, Berlin/ West 1982).

${ }^{28}$ Vgl. Helas, Autorenkollektiv 1955/58, 120-123.

${ }^{29}$ Vgl. Berthold, Marxistisches Geschichtsbild, 86-93.

${ }^{30} \mathrm{Vgl}$. Revolutionäre deutsche Parteiprogramme (Berlin 1965) 162-190.

${ }^{31}$ Vgl. Robert Leibbrand, Zur Diskussion über den Charakter der Novemberrevolution, in: Einheit 12 (1957) 102-108; Roland Bawer, Die Einschātzung des Charakters der deutschen Novem- 
Nach längerem Meinungsstreit und gestützt auf vorliegende Forschungsergebnisse setzte sich jedoch im Vorfeld des 40.Jahrestages der Revolution die Auffassung durch, wonach die Novemberrevolution eine bürgerlich-demokratische Revolution war, die in gewissem Umfang mit proletarischen Mitteln und Methoden durchgeführt wurde ${ }^{32}$. Dieser Wertung lagen Überlegungen über die Wechselbeziehung der objektiven und subjektiven Faktoren in der Revolution und die daraus resultierende Erkenntnis zugrunde, daß zwar die objektiven Voraussetzungen für eine sozialistische Revolution gegeben waren, der entscheidende subjektive Faktor jedoch für eine sozialistische Revolution - die Existenz einer politisch reifen und massenwirksamen revolutionären marxistischen Partei - zu jener Zeit in Deutschland nicht vorhanden war.

Hinsichtlich der Rolle der Räte bestand das Problem darin zu klären, ob und in welchem Maße die Räte im Interesse der Revolution oder der Konterrevolution wirksam geworden sind ${ }^{33}$. Forschungsorientierend wirkten die Forderungen nach konkrethistorischer Untersuchung der Funktion und Tätigkeit der Rāte vor und während der Novemberrevolution in den einzelnen Orten und Gebieten Deutschlands sowie eine dementsprechende, differenzierte Wertung der Rāte. Die erste, auf neuen Forschungen beruhende Monographie von Hans Beyer „Von der Novemberrevolution zur Räterepublik in München“"34 und weitere Einzeluntersuchungen ${ }^{35}$ erwiesen die Tragfãhigkeit dieses Konzepts.

Drittens - Das Verhāltnis von deutscher Geschichte und Geschichte der deutschen Arbeiterbewegung. Nach Grundsatzdiskussionen in der ersten Hālfte der 50er Jahre über den Stellenwert der Geschichte der Arbeiterbewegung in der allgemeinen und deutschen Geschichte kam es in der DDR-Geschichtswissenschaft in der zweiten Hälfte des Jahrzehnts zu einer verstärkten Hinwendung zur Geschichte der Arbeiterbewegung, als deren Ergebnis sich dieser in der nichtmarxistischen Geschichtsschreibung bis dahin weitgehend ignorierte oder arg vernachlässigte Bereich als eigenstāndige Teildisziplin herausbildete ${ }^{36}$. Lag das Schwergewicht der Forschungen zunächst bei Themen des 19 . Jahrhunderts ${ }^{37}$, so wurden nun auch zunehmend die Perioden des

berrevolution von 1918 bis 1919, in: ZfG 6 (1958) 134-142; Albert Schreiner, Auswirkungen der Großen Sozialistischen Oktoberrevolution auf Deutschland vor und während der Novemberrevolution, in: Ebenda, 29-37.

${ }^{32}$ Vgl. Walter Ulbricht, Über den Charakter der Novemberrevolution, in: ZfG 6 (1958) 717-729; Walter Nimtz, Uber den Charakter der Novemberrevolution von 1918/1919 in Deutschland, in: Ebenda, 687-715.

${ }^{33}$ Vgl. Karl Fugger, Zur Lehrbuch-Disposition (1918-1945), in: ZfG 3 (1955) 271-275; Beiträge von Walter Kleen, Marion Einhorn und Wolfgang Schumann in: ZfG 4 (1956) 326-331, 738-750, 964-989; sowie von S. K. Eggert, Rudolf Lindau und Wermer Raase in: Die Oktoberrevolution und Deutschland (Berlin 1958) 123-138, 210-214.

${ }^{34}$ (Berlin 1957).

${ }^{35}$ Vgl. Lothar Bertbold, Helmut Neef, Militarismus und Opportunismus gegen die Novemberrevolution (Berlin 1958); Hellmut Kolbe, Sturmtage (Leipzig-Jena 1958); Zum 40. Jahrestag der deutschen Novemberrevolution, Sonderheft der ZfG 6 (1958), im folgenden zitiert: Novemberrevolution.

${ }^{36} \mathrm{Vgl}$. Seiler, Geschichte der Arbeiterbewegung.

${ }^{37}$ Vgl. Walter Scbmidt, Carola Seiler, Die revolutionäre deutsche Sozialdemokratie in der Geschichtswissenschaft der DDR, in: BzG 26 (1984) 750-760. 
20.Jahrhunderts einbezogen, wobei für die Zeit nach 1918 die Geschichte der KPD im Mittelpunkt der Aufmerksamkeit stand. Dabei konnten frühere Tendenzen einer eingeengten Sicht auf das innerparteiliche Geschehen überwunden werden; die Entwicklung der KPD wurde im gesamtgesellschaftlichen Rahmen zu erfassen versucht und die gemeinsamen Traditionen mit den nichtrevolutionären Teilen der Arbeiterbewegung wurden deutlicher markiert ${ }^{38}$.

Zum Verhältnis von deutscher Geschichte und Geschichte der deutschen Arbeiterbewegung hatte Alfred Meusel schon auf der erwähnten Historikertagung 1952 betont, daß die Arbeiterbewegung einen integralen Bestandteil der deutschen Geschichte bilde und vieles ohne diese gar nicht erklärt werden könne. Zugleich warnte er vor der Tendenz, „das Pendel gar zu weit nach der anderen Seite ausschlagen zu lassen und die deutsche Geschichte gewissermaßen als eine Beilage zur Geschichte der deutschen Arbeiterbewegung zu behandeln. Das ist selbstverständlich auch verfehlt. Eine Geschichte Deutschlands ohne die Geschichte der Arbeiterbewegung ist ebenso wenig bzw. ebenso viel wert wie eine Geschichte der deutschen Arbeiterbewegung ohne die Geschichte Deutschlands. “39

Zwei Gegebenheiten begünstigten, das Verhältnis von deutscher Geschichte und Geschichte der deutschen Arbeiterbewegung gründlicher zu erfassen: Zum einen die im Laufe der 50er Jahre entwickelte und in den 60er Jahren weiterwirkende ,nationale Grundkonzeption der Arbeiterklasse“. Sie enthielt die Leitlinien der DDR-Politik zur nationalen Problematik, die auf die Schaffung eines einheitlichen demokratischen Deutschland zielten ${ }^{40}$. Den Grundtenor dieser Konzeption bildete jedoch nicht das "Gesamtdeutsche" schlechthin, sondern der soziale Inhalt der nationalen Frage, was bedeutete, daß ein einheitlicher deutscher Nationalstaat nur auf antifaschistisch-demokratischer Grundlage unter dem maßgeblichen politischen Einfluß der werktätigen Klassen und Schichten denkbar war. In der historiographischen Umsetzung folgte daraus, daß die Geschichte der Arbeiterbewegung nicht schlechthin als ein Teilbereich, sondern als Kernstück der deutschen Geschichte des 19. und 20.Jahrhunderts zu behandeln war. Das stellte neue theoretisch-methodologische und praktische Anforderungen an alle Bereiche der Geschichtswissenschaft ${ }^{41}$.

${ }^{38}$ Vgl. Die Märzkämpfe 1921 (Berlin 1956); Wilhelm Ersil, Die revolutionāre Massenbewegung der deutschen Arbeiterklasse gegen die Regierung Cuno (Phil. Diss., Berlin 1956); Lothar Berthold, Das Programm der KPD zur nationalen und sozialen Befreiung des deutschen Volkes vom August 1930 (Berlin 1956); Wolfgang Jonas, Das Leben der Mansfeld-Arbeiter 1924-1945 (Berlin 1957); Raimund Wagner, Die Arbeiterbewegung in Sachsen im Jahre 1923 (Phil. Diss., Berlin 1958); Heinz Karl, Die deutsche Arbeiterklasse im Kampf um die Enteignung der Fürsten $(1925 / 26)$ (Berlin 1957).

${ }^{39}$ Alfred Meusel, Die wissenschaftliche Auffassung der deutschen Geschichte, in: Nachlaß Meusel, Bd. 1/1, 1 (zitiert bei Heinz, DDR-Geschichtswissenschaft 1950/52, 256).

${ }^{40} \mathrm{Vgl}$. Leo Stern, Zur Nationalen Grundkonzeption der deutschen Arbeiterklasse im Kampf um die Lösung der Lebensfrage der deutschen Nation (Berlin 1961); Die geschichtliche Aufgabe der DDR und die Zukunft Deutschlands, in: ZfG 10 (1962) 758-786.

${ }^{41} \mathrm{Vgl}$. Rolf Rudolph, Die nationale Verantwortung der Historiker in der DDR, in: ZfG 10 (1962) 253-285; Lothar Bertbold, Zur Geschichte der nationalen Konzeption der deutschen Arbeiter- 
Zum anderen wurde die Bewältigung dieser Anforderungen wesentlich forciert durch das Vorhaben einer Gesamtdarstellung zur Geschichte der deutschen Arbeiterbewegung. Dazu wurde im September 1958 eine vom Zentralkomitee der SED berufene Arbeitsgruppe gebildet, der führende Repräsentanten der revolutionären Arbeiterbewegung und Historiker angehörten ${ }^{42}$. Gestützt auf die bis dahin erzielten Forschungsergebnisse ${ }^{43}$, legte sie 1963 im Ergebnis ihrer mehrjährigen Tätigkeit einen „Grundriß zur Geschichte der deutschen Arbeiterbewegung“" vor ${ }^{44}$. Als förderlich erwies sich das Erscheinen der 1959 gegründeten fachspezifischen Zeitschrift „Beiträge zur Geschichte der deutschen Arbeiterbewegung“ (ab 1969 „Beiträge zur Geschichte der Arbeiterbewegung“).

Der „Grundriß“ bildete den konzeptionellen Leitfaden für die unmittelbar nach seinem Erscheinen in Angriff genommene und 1966 veröffentlichte achtbändige „Geschichte der deutschen Arbeiterbewegung“, mit der erstmals eine wissenschaftliche Gesamtdarstellung dieser Bewegung von ihren Anfängen bis in die Gegenwart vorgelegt wurde. Von der großen Bedeutung, die dem Zeitraum von 1917 bis 1945 beigemessen wurde, zeugte die Tatsache, daß dieser Periode allein drei Bände gewidmet waren ${ }^{45}$. Mit diesem Werk wurde die beim Entstehen des mehrbāndigen „Lehrbuch der Geschichte des deutschen Volkes“ begründete Tradition wissenschaftlicher Gemeinschaftsarbeit in der DDR-Historiographie in einer noch intensiveren und auch produktiveren Weise fortgesetzt ${ }^{46}$.

Viertens - Zum Stellenwert der deutschen Geschichte von 1933 bis 1945. Ein klares Bild vom Faschismus, insbesondere über seine sozialen Wurzeln und sein Wesen zu vermitteln, aber zugleich auch seine Gegenkräfte deutlich hervortreten zu lassen, hatte für die politische Identität der DDR und für die Bewāltigung der von ihr in Angriff genommenen revolutionāren Umgestaltungen konstitutive Bedeutung. Die Grundlage für eine leistungsfähige marxistische Faschismusforschung wurde in der DDR im Laufe der 50er Jahre gelegt, und zwar von Historikern, die selbst aktiv gegen den Faschismus gekämpft hatten: Walter Bartel, Ernst Engelberg, Jürgen Kuczynski, Rudolf Lindau, Alfred Meusel, Erich Paterna, Albert Schreiner und Leo Stern. Die breiteste Ausstrahlung erlangte dabei Bartels Monographie „Deutschland in der Zeit der faschistischen Diktatur 1933-1945"47 als erste Gesamtdarstellung in der DDR zu diesem Thema. Sie war als Lehrbrief für das Fernstudium der Geschichtslehrer entstanden. Hierin zeigte sich die stimulierende Wirkung von Lehr- und Ausbildungsbedürfnissen für die Forschung.

klasse, in: Ebenda, 1005-1016; Ernst Engelberg, Probleme des nationalen Geschichtsbildes der deutschen Arbeiterklasse, in: Ebenda, Sonderheft (1962) 7-49.

${ }^{42}$ Vgl. Dokumente, Bd. VII (Berlin 1961) 353; Novemberrevolution, 54.

${ }^{43} \mathrm{Vgl}$. Historische Forschungen in der DDR, in: Sonderheft der ZfG 8 (1960) 201-457, Sonderband der ZfG 18 (1970) 380-650.

4 (Berlin 1963).

${ }^{45}$ Bd. 3 von 1917 bis 1923; Bd. 4 von 1924 bis Januar 1933; Bd. 5 von Januar 1933 bis Mai 1945.

${ }^{46}$ An der Ausarbeitung der Bände 3-5 waren als Autoren beteiligt: Lothar Berthold, Ernst Diehl, Günter Hortzschansky, Bruno Löwel, Walter Nimtz, Wolfgang Schumann, Walter Wimmer.

${ }^{47}$ (Berlin 1956). 
Eine neue Qualität erlangten die Forschungen über den Zeitraum von 1917/18 bis 1945 in der DDR im Úbergang zu den 60er Jabren. Zu den bereits ausgewiesenen Historikern kamen nunmehr Nachwuchskräfte hinzu, darunter Karl Drechsler, Klaus Drobisch, Dietrich Eichholtz, Gerhard Förster, Kurt Gossweiler, Gerhard Hass, Günter Hortzschansky, Heinz Kühnrich, Klaus Mammach, Kurt Petzold, Wolfgang Schumann, Walter Wimmer und andere. Als besonders forschungsstimulierend erwiesen sich die internationalen Konferenzen zu den Themen „Der deutsche Imperialismus und der zweite Weltkrieg“ (1959) ${ }^{48}$ sowie „Die Barbarei - extremster Ausdruck der Monopolherrschaft in Deutschland" (1961) ${ }^{49}$.

Von Beginn an verfolgte die Forschung zwei Grundlinien: Zum einen die ökonomische und politische Rolle der Monopolbourgeoisie am Vorabend und während der faschistischen Diktatur, insbesondere bei der Vorbereitung, Auslösung und Führung des Krieges sowie zum anderen der antifaschistische Widerstand, vor allem das Wirken der KPD.

Als übergreifend tragfähiges Untersuchungskonzept hinsichtlich der Rolle der Monopolbourgeoisie in der Gesamtperiode von 1917 bis 1945 erwies sich die Theorie des staatsmonopolistischen Kapitalismus, die zu Beginn der 60er Jahre in interdisziplinārer Zusammenarbeit verschiedener gesellschaftswissenschaftlicher Fächer weiterentwickelt worden war ${ }^{50}$. Verbunden mit der von Georgi Dimitroff herkommenden klassischen Wesensbestimmung des Faschismus an der Macht, konnte mit Hilfe dieser Theorie vor allem die gesellschaftliche Spezifik des Faschismus wie auch sein historischer Platz im Geschichtsprozeß des 20. Jahrhunderts genauer bestimmt werden.

Das Hauptaugenmerk galt der Beziehung von Ökonomie und Politik, von Monopolen und Staat im Faschismus. Gleichwohl wurde dabei der relativen Eigenständigkeit des faschistischen Staates und seiner Machtorgane wie auch der faschistischen Parteien und Bewegungen noch nicht genügend Rechnung getragen. Als hemmender Faktor erwies sich weiterhin das Fehlen von vergleichenden Forschungen zur Faschismusproblematik in anderen Ländern. (Trotz wesentlicher Fortschritte auf diesem Gebiet konnte dieser Mangel bis heute noch nicht gänzlich behoben werden.) Reprāsentativ für den damaligen Erkenntnisstand war der Protokollband „Monopole und Staat in Deutschland 1917-1945" 51 , der die bis dahin erzielten Forschungsergebnisse zusammenfaßte.

Von den Einzelforschungen aus der ersten Hälfte der 60er Jahre sind weiterhin Themen hervorzuheben wie die Röhm-Affäre ${ }^{52}$, die Rolle und Entwicklung der fa-

\footnotetext{
${ }^{48}$ Der deutsche Imperialismus und der zweite Weltkrieg, Bde. 1-5 (Berlin 1960-1962), im folgenden zitiert: Imperialismus.

${ }^{49}$ Beitrag von Jürgen Kuczynski und Konferenzbericht in: ZfG 9 (1961) 1494-1509, 1632-1638.

so Vgl. Jürgen Kuczynski, Studien zur Geschichte des staatsmonopolistischen Kapitalismus in Deutschland 1918 bis 1945 (Studien zur Geschichte der Lage der Arbeiter unter dem Kapitalismus 16, Berlin 1963); Imperialismus heute (Berlin 1965) 11-72.

${ }^{51}$ Protokoll der 2. Tagung der Fachgruppe Geschichte der neuesten Zeit 1917-1945 (Berlin 1966).

${ }^{32}$ Kurt Gossweiler, Die Rolle des Monopolkapitals bei der Herbeiführung der Röhm-Affäre (30. Juni 1934) (Phil. Diss., Berlin 1963); Nachdruck der Diss.: (Köln 1983).
} 
schistischen Konzentrationslager 1933 bis $1945^{53}$, Probleme der Kriegswirtschaft im faschistischen Deutschland ${ }^{54}$ sowie der Weg der deutschen Sozialdemokratie von 1933 bis $1946^{55}$. Es erschien auch die erste von DDR-Historikern verfaßte Úberblicksdarstellung zur Geschichte des Zweiten Weltkriegs ${ }^{56}$.

Die Erforschung des antifaschistischen Widerstandes entfaltete sich als Pendant der Faschismusforschung zeitlich und personell ähnlich wie diese (bis Mitte der 50er Jahre vorwiegend Arbeiten von Teilnehmern des Widerstandes, ab Mitte der 50er Jahre Wirksamwerden der neuausgebildeten Historikergeneration). Aus naheliegenden Gründen konzentrierten sich die Untersuchungen bis in die 60er Jahre hinein auf den antifaschistischen Kampf der KPD ${ }^{57}$. Das bedeutete jedoch zu keinem Zeitpunkt, daß die Widerstandsproblematik darauf reduziert worden wäre. Es ging stets um zwei Fragenkomplexe: Um die dominierende Kraft im Widerstand zum einen, aber zugleich um die Breite dieses Kampfes, um die Frage nach den Gleichgesinnten, zum anderen; die Aufmerksamkeit galt letztlich - und das im aktuellen DDR-Interesse - der Bündnisproblematik.

Eine andere Seite der Angelegenheit betrifft die konkrethistorische Umsetzung dieser grundlegenden Erkenntnisse in den einzelnen Entwicklungsabschnitten der DDRGeschichtswissenschaft. Aus heutiger Sicht sind hierbei in den 50er und 60er Jahren einige Unvollkommenheiten festzustellen. Dazu zählen vor allem die unzureichende Berücksichtigung des Widerstandes außerhalb der Arbeiterbewegung und insbesondere die undifferenzierte, vorwiegend negative Bewertung der Kräfte des 20. Juli 1944. Allerdings gab es hierzu in den konzeptionellen Debatten schon in den 50er Jahren mahnende Stimmen, die auf positive Tendenzen innerhalb dieser Kräfte hinwiesen

\footnotetext{
53 Heinz Kübnrich, Der KZ-Staat (Berlin 1960).

s4 Horst Hemberger, Einige Fragen der Kriegswirtschaft des faschistischen deutschen Imperialismus (Phil. Diss., Berlin 1960); Wolfgang Habn, Lothar Kruss, Elektrokonzerne - Rüstungskonzerne (Berlin 1961); Wolfgang Schumann, Das Kriegsprogramm des Zeiss-Konzerns, in: ZfG 11 (1963) 704-724; Dietrich Eichboltz, Probleme einer Wirtschaftsgeschichte des Faschismus in Deutschland, in: Jahrbuch für Wirtschaftsgeschichte (Berlin 1963).

5s Heinz Niemann, Otto Findeisen, Dieter Lange, SPD und Hitlerfaschismus. Der Weg der deutschen Sozialdemokratie vom 23. Januar 1933 bis zum 21. April 1946 (Phil. Diss., Berlin 1965); Neue Probleme der Geschichte der deutschen Arbeiterbewegung in Forschung und Lehre (Berlin 1965).

36 Gerbard Förster, Heinz Helmert, Helmut Scbnitter, Der zweite Weltkrieg (Leipzig 1962).

${ }^{57}$ Charakteristische Beispiele sind: Otto Winzer, Zwölf Jahre Kampf gegen Faschismus und Krieg (Berlin 1955); Walter Bartel, Probleme des antifaschistischen Widerstandskampfes in Deutschland, in: ZfG 6 (1958) 999-1016; Wilhelm Webling, Das Konzept der Kommunistischen Partei Deutschlands gegen die faschistische Diktatur des deutschen Imperialismus in den Jahren von 1933-1935 (Phil. Diss., Berlin 1960); Helene Roggenbuck, Der Widerstandskampf der illegalen KPD während des zweiten Weltkrieges in den wichtigsten Zügen und an den Schwerpunkten der inneren Front (Phil. Diss., Berlin 1961); Manfred Weißbecker, Die Kommunistische Partei Deutschlands im Kampf gegen die faschistische Diktatur in Thüringen 1933-1935 (Phil. Diss., Jena 1962); Hans-Jürgen Friderici, Die Entwicklung der Strategie und Taktik der KPD und der antifaschistische Widerstandskampf in Oberschlesien (1933-1939) (Phil. Habil., Leipzig 1965); Karl Heinz Biernat, Das Ringen der KPD um die antifaschistische Einheitsfront der Arbeiterklasse und die antifaschistische Volksfront 1933 bis 1945, in: Geschichtsunterricht und Staatsbürgerkunde 8 (1966) 335-345.
} 
und eine differenziertere Betrachtungsweise forderten ${ }^{58}$. Wenn diese kritischen Einwände erst in der zweiten Hālfte der 60er Jahre zu den angestrebten Korrekturen führten ${ }^{59}$, dann hat das sehr unterschiedliche Gründe; vor allem lag das am unzureichenden Forschungsstand, aber auch an einigen Begleiterscheinungen der konfrontativen Auseinandersetzung mit restaurativen Auffassungen in der Bundesrepublik. Dort versuchten die tonangebenden Historiker einerseits den Widerstand der Arbeiterbewegung, insbesondere den der Kommunisten, abzuwerten oder gar als antinational hinzustellen und andererseits die konservativen Kräfte des 20.Juli 1944 - auch undifferenziert - zu verklären und zur einzigen ernstzunehmenden Widerstandskraft gegen den Faschismus zu erheben ${ }^{60}$. Die kritische Auseinandersetzung mit diesen Tendenzen und darüber hinaus generell mit den Darstellungen der bürgerlichen Historiographie zur deutschen Geschichte, insbesondere über den Zeitraum von 1917/18 bis 1945 spielte eine bedeutende Rolle im Selbstverstāndigungsprozeß der marxistischen Geschichtswissenschaft in der DDR ${ }^{61}$.

In der zweiten Hälfte der 60er Jahre erreichte die Geschichtswissenschaft der DDR ihre Kulmination im Entwicklungsprozeß nach 1945. Davon zeugten für jedermann sichtbar der Abschluß der beiden Gesamtdarstellungen. Nachdem jene zur „Geschichte der deutschen Arbeiterbewegung in acht Bänden“ bereits 1966 veröffentlicht werden konnte, erschienen bis 1969 die restlichen drei Bānde des „Lehrbuch der deutschen Geschichte (Beiträge) “ zur Periode von 1917/18 bis $1945^{62}$. Damit konnten die Historiker der DDR zum ersten Mal eine alle geschichtlichen Perioden umfassende Darstellung der deutschen Geschichte auf der Grundlage des historischen Materialismus vorlegen. War dieses Werk hauptsāchlich für Lehrende und Studierende an Universitäten und Hochschulen gedacht, so wandte sich die von Joachim Streisand

${ }^{38}$ Fischer, Autorenkollektiv 1952/55, 148; Hans Dress, Fortschrittliche und reaktionāre Tendenzen in den Reformplānen des Kreisauer Kreises, in: Imperialismus, Bd. 4.

${ }^{39}$ Kurt Finker, Stauffenberg und der 20. Juli 1944 (Berlin 1967).

${ }^{60}$ Richtungsweisend dafür waren: Gerbard Ritter, Carl Goerdeler und die deutsche Widerstandsbewegung (Stuttgart 1954); Hans Rothfels, Die deutsche Opposition gegen Hitler (Krefeld 1949). Einen Einblick in die bundesdeutsche historiographische Widerstandsliteratur bis Mitte der 60er Jahre vermitteln: Thilo Vogelsang, Die Widerstandsbewegung und ihre Problematik in der zeitgeschichtlichen Darstellung, in: Das Parlament, Beilage 28/65 vom 14.Juli 1965; Friedrich Zipfel, Die Bedeutung der Widerstandsforschung für die allgemeine zeitgeschichtliche Forschung, in: Ebenda.

${ }^{61}$ Vgl. Probleme der Geschichte des zweiten Weltkrieges, Bd. II (Berlin 1958); Werner Berthold, "... großhungern und gehorchen.“ Zur Entstehung und politischen Funktion der Geschichtsideologie des westdeutschen Imperialismus untersucht am Beispiel von Gerhard Ritter und Friedrich Meinecke (Berlin 1960); Gerhard Lozek, Horst Syrbe, Geschichtsschreibung contra Geschichte (Berlin 1964).

${ }^{62}$ Wolfgang Ruge, Deutschland von 1917 bis 1933 (Berlin 1967); Erich Paterna, Werner Fischer, Kurt Gossweiler, Gertraud Markus, Kurt Pätzold, Deutschland von 1933 bis 1939 (Berlin 1969); Wolfgang Bleyer, Karl Drecbsler, Gerbard Förster, Gerbart Hass, Deutschland von 1939 bis 1945 (Berlin 1969). Die Hauptergebnisse dieser Bānde waren in den Bd. 3 einer repräsentativen „Deutschen Geschichte in drei Bänden" eingegangen (Berlin 1968). 
verfaßte „Deutsche Geschichte in einem Band ${ }^{463}$, an einen breiteren Leserkreis. Der Band berücksichtigte bereits über das Lehrbuch hinausgehende Erkenntnisse.

Parallel zu den Gesamtdarstellungen erschienen zahlreiche Monographien zur deutschen Geschichte von 1917/18 bis 1945, die wesentliche Bausteine für das marxistische Geschichtsverständnis darstellten ${ }^{64}$. Langfristige Wirkungen in diese Richtung gingen nicht zuletzt von Handbüchern und Nachschlagewerken aus, die zumeist die ganze deutsche Geschichte umfaßten, aber auch vielfältige Erkenntnisse zu dem hier interessierenden Geschichtsabschnitt vermittelten ${ }^{65}$.

Die marxistische Geschichtswissenschaft der DDR hatte bis Ende der 60er Jahre ein breites und solides Forschungsfundament geschaffen, von dem aus neue Aufgaben in Angriff genommen werden konnten. Unmittelbar an die erzielten Ergebnisse und gewonnenen Erfahrungen kritisch anknüpfend, kam es in der Folgezeit vor allem zu einem weiteren Klärungsprozeß hinsichtlich der Wechselbeziehung von Nationalund Weltgeschichte ${ }^{66}$, was zu einer beachtlichen Ausweitung der Forschungsfelder und -themen führte.

${ }^{63}$ (Berlin 1968). Eine bearbeitete Fassung des Buches erschien in der Bundesrepublik unter dem Titel: Deutsche Geschichte von den Anfängen bis zur Gegenwart (Köln 1972).

${ }^{64}$ Vgl. Jürgen Kuczynski, Darstellung der Lage der Arbeiter in Deutschland von 1933 bis 1945 (Die Geschichte der Lage der Arbeiter unter dem Kapitalismus 6, Berlin 1964); ders, Darstellung der Lage der Arbeiter in Deutschland von 1917/18 bis 1932/33 (Ebenda 5, Berlin 1966); Siegfried Vietzke, Heinz Woblgemuth, Deutschland und die deutsche Arbeiterbewegung in der Zeit der Weimarer Republik (Berlin 1966); Wolfgang Ruge, Weimar - Republik auf Zeit (Berlin 1969); Dietrich Eicbboltz, Geschichte der deutschen Kriegswirtschaft 1939-1945, Bd. I: 1939-1941 (Berlin 1969).

${ }^{65} \mathrm{Vgl}$. Kleine Enzyklopãdie. Deutsche Geschichte von den Anfāngen bis 1945 (Leipzig 1965); Deutsche Geschichte in Daten (Berlin 1967); Die bürgerlichen Parteien in Deutschland (Leipzig 1968); Biographisches Lexikon zur Geschichte der deutschen Arbeiterbewegung (Berlin 1969); Biographisches Lexikon zur deutschen Geschichte (Berlin 1971); Sachwörterbuch zur deutschen Geschichte, 2 Bde. (Berlin 1969, 1970).

${ }^{66}$ Wesentliche Impulse gingen vom V. Historikerkongreß der DDR aus, der im Dezember 1972 in Dresden stattfand und dem Thema „Die Geschichte des deutschen Volkes im welthistorischen Prozei ${ }^{\prime \prime}$ gewidmet war. Referate und Berichte des Kongresses sind veröffentlicht in: ZfG 20 (1972) 1228-1284 und 21 (1973) 441-454. 
